\title{
Numerical analysis of coupled thermo-hydraulic problems in
} \section{geotechnical engineering}

\author{
W. Cui ${ }^{1}$, K.A. Gawecka ${ }^{2}$, D.M. Potts ${ }^{3}$, D.M.G. Taborda ${ }^{4}$ and L. Zdravković ${ }^{5}$ \\ Civil and Environmental Engineering, Imperial College London, London, SW7 2AZ, United Kingdom \\ ${ }^{1}$ w.cuil1@imperial.ac.uk \\ ${ }^{2}$ klementyna.gawecka09@imperial.ac.uk (corresponding author) \\ 3 d.potts@imperial.ac.uk \\ ${ }^{4}$ d.taborda@imperial.ac.uk \\ ${ }^{5}$ l.zdravkovic@imperial.ac.uk
}

\begin{abstract}
Ground sources energy systems, such as open-loop systems, have been widely employed in recent years due to their economic and environmental benefits compared to conventional heating and cooling systems. Numerical modelling of such geothermal system requires solving a coupled thermohydraulic problem which is characterised by a convection-dominated heat transfer which can be challenging for the Galerkin finite element method (GFEM). This paper first presents the coupled thermo-hydraulic governing formulation as well as the coupled thermo-hydraulic boundary condition, which can be implemented into a finite element software. Subsequently, the stability condition of the adopted time marching scheme for coupled thermo-hydraulic analysis is established analytically. The behaviour of highly convective problems is then investigated via a series of analyses where convective heat transfer along a soil bar is simulated, with recommendations on the choice of an adequate discretisation with different boundary conditions being provided to avoid oscillatory solutions. Finally, the conclusions from the analytical and numerical studies are applied to the simulation of a boundary value problem involving an open-loop system, with the results showing good agreement with an approximate solution. The main objective of this paper is to demonstrate that the GFEM is capable of dealing with highly convective geotechnical problems.
\end{abstract}

Keywords: finite element analysis; coupled analysis; convective heat transfer; open-loop systems

\section{Introduction}

Diminishing fossil fuel reserves and growing energy demand have led to an increased interest, as well as technological advances, in the renewable energy sector. In recent years, geotechnical engineering has experienced challenges associated with utilising shallow geothermal energy - the energy stored in the ground up to depths of $300 \mathrm{~m} \mathrm{[1]} \mathrm{-} \mathrm{as} \mathrm{ground} \mathrm{source} \mathrm{energy} \mathrm{systems} \mathrm{are} \mathrm{becoming} \mathrm{increasingly}$ popular.

These geothermal systems are used to extract and/or inject heat from and into the ground by either directly abstracting water from an aquifer through a well and returning it through another well located at a distance (open-loop systems), or pumping a fluid through a system of pipes buried in the ground or placed in buildings' foundations (closed-loop systems).

Open-loop systems can provide a higher energy yield than closed-loop systems, however, they have a higher financial risk due to running costs and a higher environmental risk associated with possible groundwater pollution [2]. Spacing of the wells is a particularly important aspect of the design of open-loop systems. If the wells are too close, the thermal plume of cold or warm water from the 
1 injection well may reach the abstraction well and reduce the efficiency of the system [1]. This 2 phenomenon is known as thermal breakthrough.

3 To model open-loop systems, two types of numerical methods - the finite difference (FD) method and 4 the finite element (FE) method, have been adopted in the literature. Todd and Banks [3] and Gandy et scheme located in the UK. One of the most widely used finite element codes for thermo-hydraulic (TH) analysis is FEFLOW [6], which has been used by Lo Russo and Civita [7], Nam and Ooka [8], and Bridger and Allen [9], amongst others, to simulate open-loop systems. However, the details of the FE analysis, particularly in terms of the numerical method or the boundary conditions, have been the subject of limited discussion.

Open-loop ground source energy systems are characterised by convection-dominated heat transfer and can be modelled numerically as coupled thermo-hydraulic problems. To obtain the solution to this complex problem in a finite element program, it is necessary to develop a formulation which couples the governing equations for groundwater flow and ground heat transfer, as well as appropriate boundary conditions. As the processes of both pore water flow and heat transfer are time dependent, a stable time marching scheme is also required. However, it has been noted in the literature that, when the FE method is used to simulate a highly convective problem, the extensively used Galerkin finite element method (GFEM) often produces numerical oscillations, if the mesh is too coarse $[10,11]$. To eliminate this problem, the use of upwind finite element methods, including the Petrov-Galerkin method [10, 11], the Petrov-Galerkin least square method [12], and the shock capturing method [13], is recommended. However, these methods obtain the solution by either modifying the weighting function or introducing an artificial damping which changes the physics of the problem and results in a reduction in accuracy. Diersch [6] compared some of these methods in simulations of a model test of a well doublet system and concluded that, although the upwind methods ensure a non-oscillatory solution, using GFEM can also lead to stabilised results provided the mesh and the time marching scheme are chosen adequately. Nonetheless, limited information has been provided on quantifying the effect of the mesh or the boundary conditions on the oscillations encountered when using GFEM.

In this paper, the behaviour of numerical analysis of highly convective geotechnical problems has been investigated using the Imperial College Finite Element Program - ICFEP [14], which has recently been upgraded to simulate fully coupled thermo-hydro-mechanical (THM) behaviour of porous materials. Firstly, the coupled thermo-hydraulic formulation implemented in ICFEP is validated and the need for a coupled thermo-hydraulic boundary condition is illustrated. Moreover, the stability condition of the time marching $\theta$-method, which is adopted in ICFEP for solving the heat conductive-convective equation, is studied analytically. Subsequently, studies on the behaviour of numerical solutions to highly convective problems with different boundary conditions are presented. Lastly, the resulting findings, including the obtained stability condition as well as the conclusions from the numerical studies, are applied to simulate an open-loop ground source energy system, with the predicted time to thermal breakthrough being compared to an available approximate solution.

\section{Coupled thermo-hydraulic finite element analysis}

\subsection{Governing formulation}

\subsubsection{Pore fluid flow}

The continuity equation defined by Equation ( 1 ) must be satisfied by incompressible pore fluid flow in a fully saturated porous medium, such as soil.

$$
\frac{\partial v_{x}}{\partial x}+\frac{\partial v_{y}}{\partial y}+\frac{\partial v_{z}}{\partial z}-Q^{w}=\frac{\partial \varepsilon_{v}}{\partial t}
$$


where $v_{x}, v_{y}, v_{z}$ are the components of the superficial velocity of the pore fluid in the $\mathrm{x}, \mathrm{y}$ and $\mathrm{z}$ directions, respectively, $\varepsilon_{v}$ is the volumetric strain of the soil skeleton due to stress changes, $Q^{w}$ represents any pore fluid sources and/or sinks, and $t$ is time. The seepage velocity, $\left\{v_{w}\right\}^{T}=\left\{v_{x}, v_{y}, v_{z}\right\}$, is assumed to be governed by Darcy's law, which can be written as:

$$
\left\{v_{w}\right\}=-\left[k_{w}\right]\{\nabla h\}
$$

where $\left[k_{w}\right]$ is the permeability matrix and $\nabla h$ is the gradient of the hydraulic head. In a coupled thermo-hydraulic problem, if the effect of temperature gradients on pore fluid flow through a fully saturated soil is assumed to be negligible and the soil skeleton is assumed to be rigid, Equation ( 1 ) reduces to the equation for steady state seepage, which can be expressed as:

$$
\frac{\partial v_{x}}{\partial x}+\frac{\partial v_{y}}{\partial y}+\frac{\partial v_{z}}{\partial z}=Q^{w}
$$

\subsubsection{Heat transfer}

There are three main modes of heat transfer in a fully saturated soil: conduction, convection and radiation. However, the effect of radiation is often assumed to be negligible compared to the effects of conduction and convection [15], and therefore is not taken into account in this formulation. The equation governing heat transfer is based on the law of conservation of energy, and can be written as:

$$
\frac{\partial\left(\Phi_{\theta} d V\right)}{\partial t}+\nabla \cdot\left\{Q_{\theta}\right\} d V=Q^{\theta} d V
$$

where $\Phi_{\theta}$ is the heat content of soil per unit volume, $Q_{\theta}$ is the heat flux per unit volume including heat conduction and heat convection, $Q^{\theta}$ are any heat sources/sinks and $d V$ is the volume of the soil. The heat content, $\Phi_{\theta}$, is calculated according to the proportion of different soil components. For fully saturated soils, it is defined as:

$$
\Phi_{\theta}=\left[n \rho_{w} C_{p w}+(1-n) \rho_{s} C_{p s}\right] T
$$

where $C_{p w}$ and $C_{p s}$ are the specific heat capacities of pore water and soil particles, respectively, $\rho_{w}$ and $\rho_{s}$ are the densities of pore water and soil particles, respectively, $n$ is porosity, $T$ is the temperature in Kelvin of the pore water and soil particles, which are assumed to be in thermal equilibrium.

The heat flux term, $Q_{\theta}$, in Equation ( 4 ) can be written as the sum of heat conduction, $q_{d}$, and heat convection, $q_{c}$ :

$$
\left\{Q_{\theta}\right\}=\left\{q_{d}\right\}+\left\{q_{c}\right\}
$$

with heat conduction being described by Fourier's Law:

$$
\left\{q_{d}\right\}=-\left[k_{\theta}\right]\{\nabla T\}
$$

where $\left[k_{\theta}\right]$ is the thermal conductivity matrix, while the convection term is given by:

$$
\left\{q_{c}\right\}=\rho_{w} C_{p w}\left\{v_{w}\right\} T
$$

In a coupled thermo-hydraulic analysis, if the soil is assumed to be rigid, Equation ( 4 ) reduces to:

$$
\left[n \rho_{w} C_{p w}+(1-n) \rho_{s} C_{p s}\right] \frac{\partial T}{\partial t}+\rho_{w} C_{p w}\left\{v_{w}\right\}^{\mathrm{T}}\{\nabla T\}-\nabla \cdot\left(\left[k_{\theta}\right]\{\nabla T\}\right)=Q^{\theta}
$$


1 Combining Equations ( 2 ), ( 3 ) and ( 9 ) leads to the finite element formulation for coupled thermo2 hydraulic problems. The time marching scheme of the $\theta$-method is adopted for solving the heat 3 transfer equations following a similar process to that for consolidation and seepage [14].

\section{$4 \quad 2.2$ Coupled thermo-hydraulic boundary condition}

5 To validate the coupled thermo-hydraulic finite element formulation, several analyses on a $1 \mathrm{~m}$ bar of soil with element length of $0.01 \mathrm{~m}$, subjected to one-dimensional (1D) convection-dominated heat transfer, were conducted in ICFEP using 4-noded linear quadrilateral two-dimensional (2D) elements. The geometry of the considered problem is shown in Figure 1 and the material properties used in the simulations are listed in Table 1. To include the convective heat transfer, a constant pore water pressure gradient was applied over the mesh, inducing a pore water flow with a constant velocity from left to right. The applied pore water pressure gradient was varied in this exercise in order to study the influence of different values of water velocity on heat convection. A constant temperature boundary condition of $20^{\circ} \mathrm{C}$ was applied at the left-hand side of the bar, while the initial temperature was $10{ }^{\circ} \mathrm{C}$, generating heat transfer along the bar. To ensure that the simulated phenomenon was one-dimensional, no pore water flow or heat transfer was allowed through the horizontal boundaries of the problem. The $\theta$-method was applied with the backward difference scheme $(\theta=1.0)$, while the time-step was chosen arbitrarily as $60 \mathrm{~s}$.

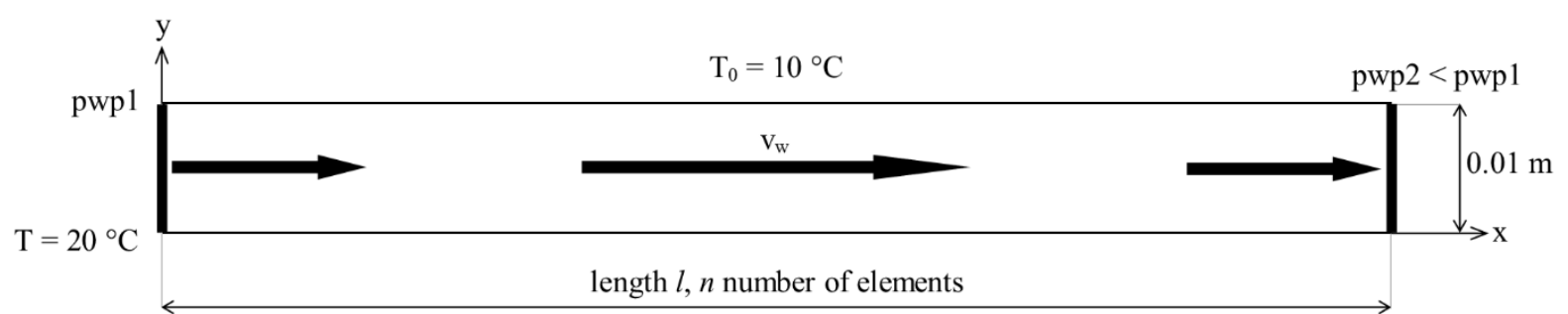

Figure 1 Geometry of the analysed problem and boundary conditions

Table 1 Material properties adopted in the heat transfer analysis

\begin{tabular}{ll}
\hline Density of solids, $\rho_{s}\left(\mathrm{~kg} / \mathrm{m}^{3}\right)$ & 2500 \\
Density of water, $\rho_{w}\left(\mathrm{~kg} / \mathrm{m}^{3}\right)$ & 1000 \\
Specific heat capacity of solids, $C_{p s}\left(\mathrm{~kJ} / \mathrm{kg} \cdot{ }^{\circ} \mathrm{C}\right)$ & 0.88 \\
Specific heat capacity of water, $C_{p w}\left(\mathrm{~kJ} / \mathrm{kg} \cdot{ }^{\circ} \mathrm{C}\right)$ & 4.19 \\
Thermal conductivity, $k_{\theta}\left(\mathrm{kJ} / \mathrm{s} \cdot \mathrm{m} \cdot{ }^{\circ} \mathrm{C}\right)$ & 0.001 \\
Porosity, $n$ & 0.23 \\
Permeability, $k_{w}(\mathrm{~m} / \mathrm{s})$ & $5.5 \times 10^{-5}$ \\
\hline
\end{tabular}

Figure 2 shows the nodal temperature distribution along the bar after $3600 \mathrm{~s}$. A sharp increase in temperature can be clearly observed at the right-hand side boundary of the soil bar, even though the heat front has not yet arrived there, as suggested by the fact that the temperature in the middle of the bar is still at its original value $\left(10^{\circ} \mathrm{C}\right)$. It should also be noted that this unrealistic increase in temperature initially started at the boundary where pore water leaves the mesh, with a higher increase in temperature being found in the analysis with a larger pore water velocity. This scenario is clearly physically impossible, and its characteristics imply that in the analyses above the pore water leaves the mesh, but the energy equivalent to the volume of water remains, generating unrealistic increases in temperature at this boundary. 


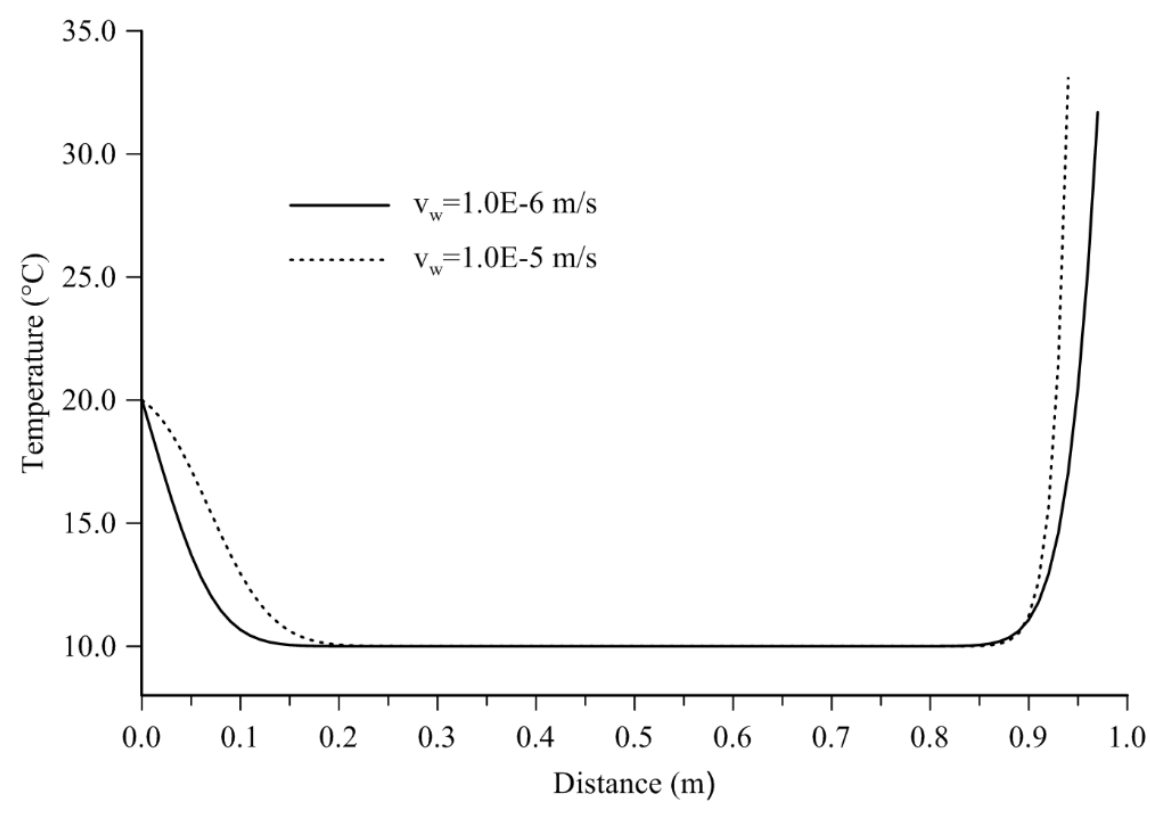

Figure 2 Temperature distribution for different fluid velocities at $t=3600 \mathrm{~s}$

3 To avoid this issue, a boundary condition was proposed and implemented into ICFEP, which prescribes a heat flux at the boundary where water leaves or enters the mesh in order to balance the change of energy associated with the water flow through the boundary. The total amount of energy associated with the flow of pore water through the boundary can be determined from the following equation:

$$
q_{\theta, C}=\int_{d \Omega} \rho_{w} C_{p w}\left\{v_{w}\right\}_{b} T_{b} d \Omega
$$

8 where $\left\{v_{w}\right\}_{b}$ represents the velocity of the pore water flowing through the boundary, $T_{b}$ is the current 9 temperature at the boundary, and $\Omega$ denotes either a line, a surface or a volume over which the 10 boundary condition is prescribed. It should be noted that the proposed boundary condition is non11 linear, with both $\left\{v_{w}\right\}_{b}$ and $T_{b}$ varying over an increment, which must be accounted for by the 12 software. Therefore, it is defined as a coupled thermo-hydraulic boundary condition.

13 The proposed coupled thermo-hydraulic boundary condition was then applied to the previously 14 described numerical analysis of 1D convection-dominated heat transfer. As a fixed temperature boundary condition was already prescribed at the left-hand side boundary of the mesh, which automatically balances the change of energy related to the volume of water flowing into the mesh, the proposed boundary condition was only prescribed at the right-hand side boundary of the bar where water flows out of the mesh. Figure 3 shows the nodal temperature distribution along the bar with the coupled thermo-hydraulic boundary condition. The sharp increase in temperature at the right-hand side boundary of the bar disappears and the numerical results with different values of water velocity obtained using ICFEP agree very well with the analytical results from van Genuchten and Alves [16]. 


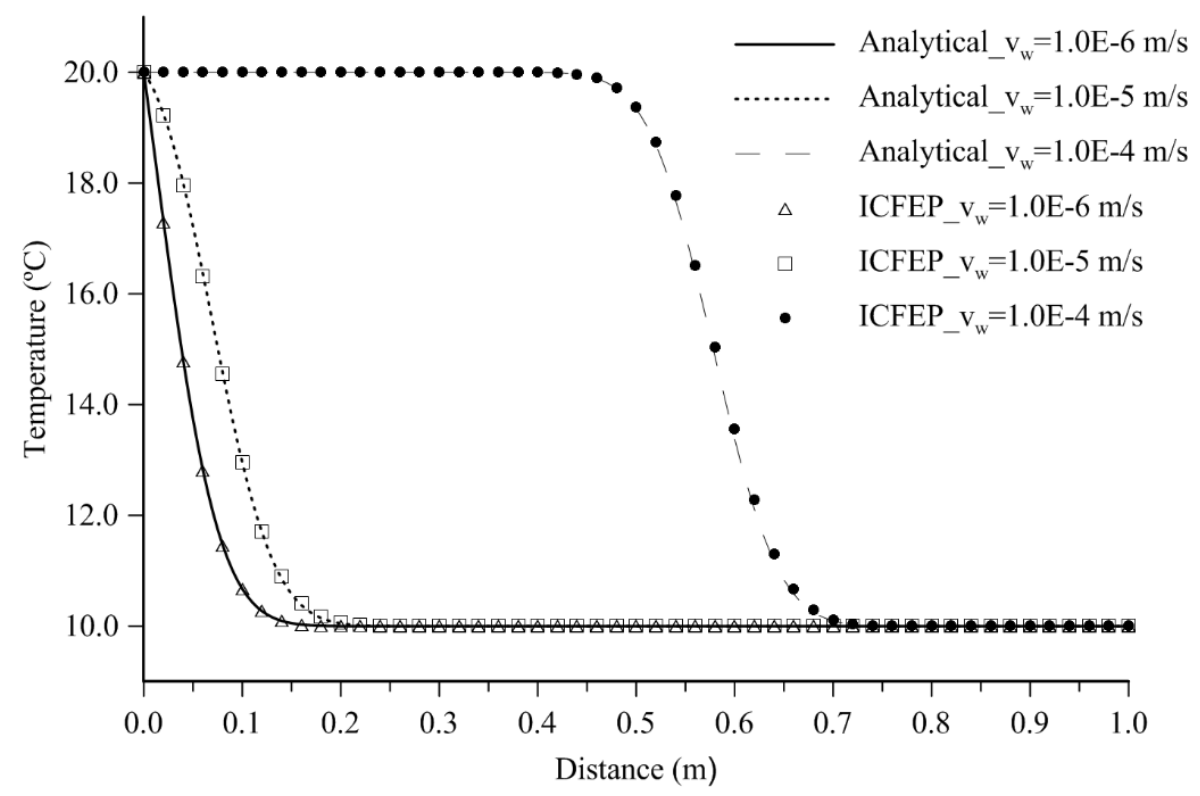

Figure 3 Comparison of analytical and numerical results of temperature distribution for different fluid velocities with the coupled thermo-hydraulic boundary condition at $t=3600 \mathrm{~s}$

43 Stability of the time integration scheme for coupled thermo5 hydraulic FE analysis

6 In the finite element analysis of coupled thermo-hydraulic problems, it is necessary to adopt a time 7 integration scheme in order to approximate the numerical solutions over a time interval $\Delta t$. One of the 8 important aspects of any time integration scheme is its stability conditions. A stable time integration 9 scheme can produce bounded results, which means that the errors between numerical solutions and the exact solutions either decrease or remain constant during subsequent steps [17]. In this research, the $\theta$-method, which has been extensively used in transient $\mathrm{FE}$ analyses, is applied to solve the governing equations for coupled thermo-hydraulic problems. The stability conditions of the $\theta$-method in solving the hydraulic equation and the thermal equation without the convection term have been investigated by Booker and Small [18] and Al-Khoury [19], respectively. To examine the stability condition of the $\theta$-method in solving the equation governing the conductive-convective heat transfer, an eigenvalue analysis was performed.

17 Considering the convective heat transfer along a 1D bar in the $\mathrm{x}$-direction without a heat source/sink, 18 Equation ( 9 ) can be simplified to:

$$
\rho C_{p} \frac{\partial T}{\partial t}+\rho_{w} C_{p w} v_{w} \frac{\partial T}{\partial x}-k_{\theta} \frac{\partial^{2} T}{\partial x^{2}}=0
$$

where $\rho$ and $C_{p}$ are the density and the specific heat capacity of the material, respectively. For soils, 20 the following expression can be adopted:

$$
\rho C_{p}=n \rho_{w} C_{p w}+(1-n) \rho_{s} C_{p s}
$$

21 Discretising Equation ( 11 ) using the Galerkin method and evaluating the obtained integrals leads to:

$$
[C] \cdot\left\{\frac{\partial T}{\partial t}\right\}+([K]+[D]) \cdot\{T\}=\{0\}
$$


where $[C]$ is the mass matrix representing heat content, and $[K]$ and $[D]$ are the matrices representing heat conduction and heat convection, respectively. According to the $\theta$-method, the following relationships can be established:

$$
\begin{gathered}
\frac{\partial T}{\partial t}=\frac{\Delta T}{\Delta t}=\frac{T_{n+1}-T_{n}}{\Delta t} \\
T=\theta T_{n+1}+(1-\theta) T_{n}
\end{gathered}
$$

where the subscripts denote the integration step number. Substituting Equations ( 14 ) and ( 15 ) into Equation ( 13 ) leads to:

$$
\left\{T_{n+1}\right\}=[B]\left\{T_{n}\right\}
$$

6 where

$$
[B]=\{[C]+\theta \Delta t([K]+[D])\}^{-1}\{[C]-(1-\theta) \Delta t([K]+[D])\}
$$

7 To ensure the time integration scheme is stable, the modulus of the eigenvalues of matrix $[B]$ should be less than or equal to 1 [18]. Moreover, it has been reported by Zienkiewicz et al. [17] that the system eigenvalues are bounded by the eigenvalues of its individual elements. For linear elements, the elemental matrices which form the matrix of $[B]$ can be written as:

$$
\begin{gathered}
{[K]^{e}=\frac{\alpha}{h}\left[\begin{array}{cc}
1 & -1 \\
-1 & 1
\end{array}\right]} \\
{[D]^{e}=\frac{\rho_{w} C_{p w} v_{w}}{2 \rho C_{p}}\left[\begin{array}{cc}
-1 & 1 \\
-1 & 1
\end{array}\right]} \\
{[C]^{e}=\frac{h}{6}\left[\begin{array}{ll}
2 & 1 \\
1 & 2
\end{array}\right]}
\end{gathered}
$$

11 where $\alpha$ is the thermal diffusivity defined as $\alpha=k_{\theta} / \rho C_{p}$, and $h$ is the element length. Substituting 12 Equations ( $18-20$ ) into Equation ( 17 ) yields the expression of the elemental matrix [B]:

$$
[B]^{e}=\frac{1}{3\left(g_{3}+2 \theta \Delta t g_{1}\right)}\left[\begin{array}{cc}
3\left(g_{3}+2 \theta \Delta t g_{1}\right)+\Delta t\left(-3 g_{1}+g_{2}\right) & \Delta t\left(3 g_{1}-g_{2}\right) \\
\Delta t\left(3 g_{1}+g_{2}\right) & 3\left(g_{3}+2 \theta \Delta t g_{1}\right)+\Delta t\left(-3 g_{1}-g_{2}\right)
\end{array}\right]
$$

14 where $g_{1}=\alpha / h, g_{2}=\rho_{w} C_{p w} \nu_{w} / 2 \rho C_{p}$ and $g_{3}=h / 6$. To obtain the eigenvalues $\lambda_{i}$ of the matrix of [B], the 15 solution to the following equation is required:

$$
|[B]-\lambda I|=0
$$

16 where $I$ is the identity matrix. Solving Equation ( 22 ) results in:

$$
\lambda_{1}=1 \text { or } \lambda_{2}=\frac{1-\frac{2 g_{1}}{g_{3}}(1-\theta) \Delta t}{1+\frac{2 g_{1}}{g_{3}} \theta \Delta t}
$$


1 As previously mentioned, the eigenvalues are required to satisfy the conditions $\left|\lambda_{i}\right| \leq 1$ to ensure that

2 the $\theta$-method is stable. Therefore, it can be concluded that:

1) For $0.5 \leq \theta \leq 1$, the algorithm is unconditionally stable,

2) For $\theta<0.5$, the algorithm is stable provided that $\Delta t \leq h^{2} / 6 \alpha(1-2 \theta)$.

Applying the same procedure to quadratic elements also results in an unconditionally stable algorithm

6 for $0.5 \leq \theta \leq 1$. It should be noted that the same stability condition applies to the hydraulic equations

$7 \quad[18]$ and, therefore, it can also be used in a coupled thermo-hydraulic analysis.

\section{FE analysis of highly convective heat transfer}

\section{$9 \quad 4.1$ Péclet number}

10 In coupled thermo-hydraulic problems, the heat flux is often characterised in terms of a Péclet number

$11(\mathrm{Pe})$, which represents the ratio between the convective and the conductive transport rates. A low

12 Péclet number represents a conduction-dominated flux, whereas a high Péclet number indicates that

13 the flux is dominated by convection. For a two-phase material, such as a saturated soil, the Péclet

14 number can be defined as:

$$
P e=\rho_{w} C_{p w} v_{w} L / k_{\theta}
$$

where $L$ is the characteristic length, which, in the context of finite element analysis, is defined to be equal to the element length in the direction of fluid flow. Therefore, with given material properties and a constant fluid velocity, a coarse mesh will result in a large Péclet number, while refining the mesh will reduce its magnitude.

\subsection{Effect of Péclet number on the solution to convective problems with prescribed temperature boundary condition}

For coupled thermo-hydraulic problems dominated by conduction, the extensively used Galerkin finite element method, which assumes that the chosen weighting function is the same as the shape function, is capable of providing bounded numerical solutions under all circumstances if the time integration scheme is stable, as shown in the previous section. However, for highly convective problems, it has been reported in the literature $[6,19]$ that the Galerkin finite element method leads to the occurrence of oscillatory solutions if the Péclet number is too large. To illustrate this issue, a series of numerical simulations of $1 \mathrm{D}$ convection-dominated heat transfer along a $10 \mathrm{~m}$ bar of soil, initially at a temperature of $10{ }^{\circ} \mathrm{C}$, was performed in ICFEP, with both linear and quadratic elements. The geometry of the problem is identical to that shown in Figure 1, extended to $10 \mathrm{~m}$, while the material properties are listed in Table 1 . Similar boundary conditions to those adopted in the example shown previously were prescribed at the boundaries of the mesh. However, in order for the results to be comparable to those presented in Al-Khoury [19], a constant temperature of $10{ }^{\circ} \mathrm{C}$ was applied at the right-hand side of the bar, instead of the coupled thermo-hydraulic boundary condition. A constant pore water velocity of $2.4 \times 10^{-5} \mathrm{~m} / \mathrm{s}$ from left to right was applied so that by varying the element size, different values of Péclet number could be obtained. For example, a mesh with 100 elements of $0.1 \mathrm{~m}$ in length results in a Péclet number of 10. 


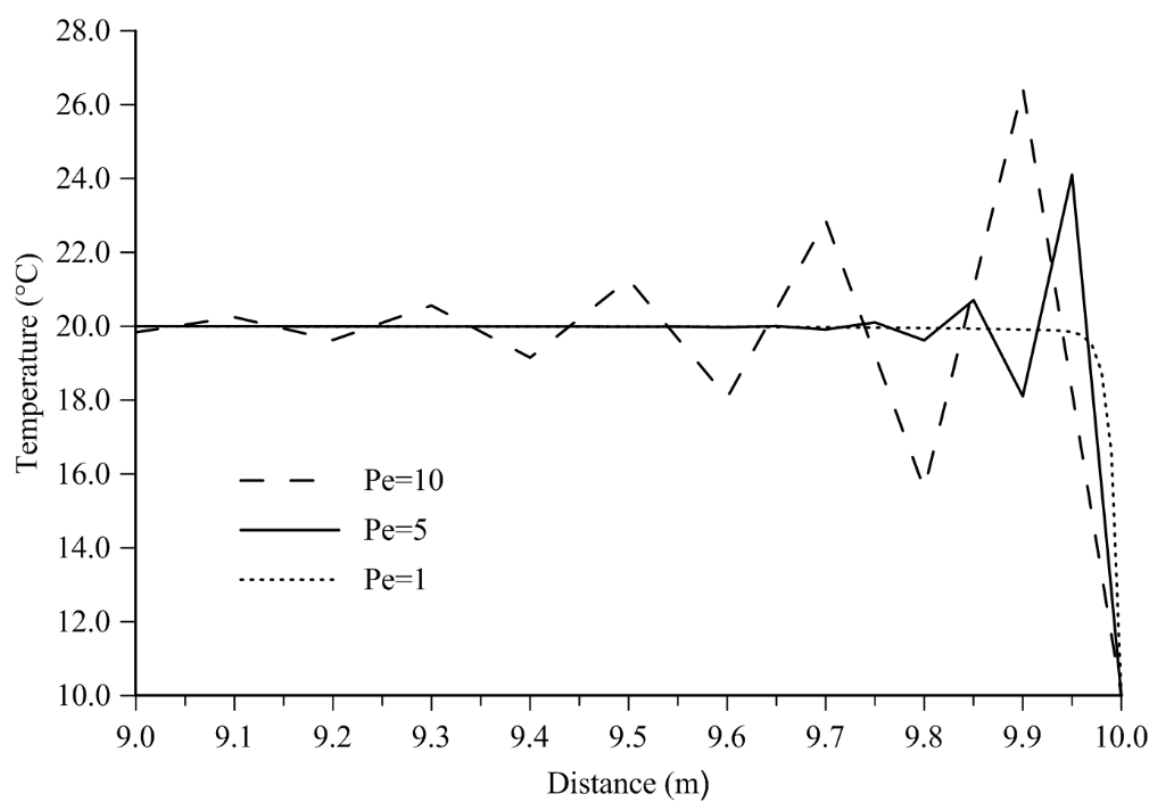

Figure 4 Temperature distribution at steady state for analyses with linear elements, prescribed temperature boundary condition and different values of Péclet number (detail of the right-hand side extremity)

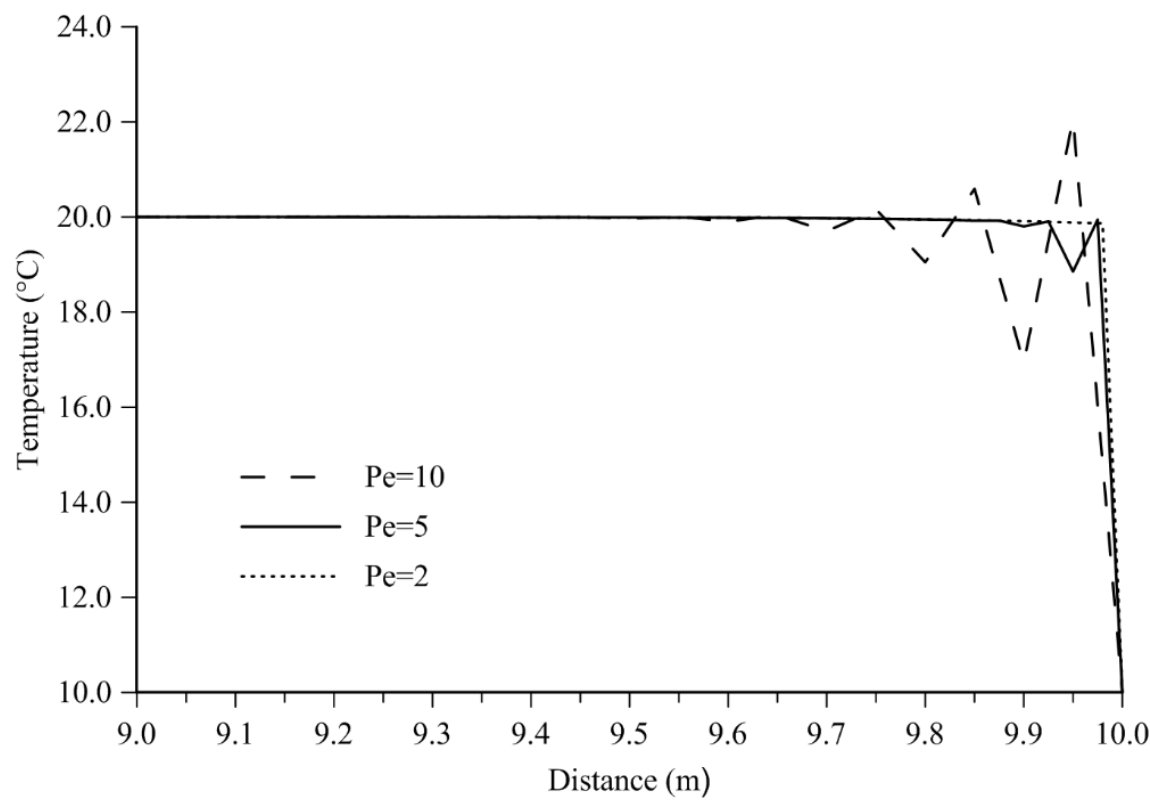

Figure 5 Temperature distribution at steady state for analyses with quadratic elements, prescribed temperature boundary condition and different values of Péclet number (detail of the right-hand side extremity)

Figures 4 and 5 show a close-up of the temperature distribution along the bar after the heat front reaches the right-hand side boundary of the mesh for analyses with different values of Péclet numbers using linear and quadratic elements, respectively. The results show an increasing amplitude of spatial oscillations with increasing Péclet number. It should be noted that the oscillations appear immediately after the heat front reaches the end of the mesh and increase until steady state is reached. Similar behaviour has also been observed by Donea and Huerta [20] and Al-Khoury [19]. It should be noted that quadratic elements experience smaller oscillations than linear elements for identical values of the Péclet number. This suggests that, in effect, the amplitude of oscillations depends on the distance between two adjacent nodes, which in quadratic elements is, naturally, half of that in linear elements. This is further demonstrated by the non-oscillatory condition for the two types of elements, which was established by complementing the study above with various analyses spanning a wide range of Péclet 
numbers. As expected, the results showed that for highly convective heat transfer with prescribed temperature boundary conditions, non-oscillatory solutions were obtained for $P e \leq 1$ for linear elements and $P e \leq 2$ for quadratic elements, reflecting the change in distance between adjacent nodes. The temperature profiles for these two cases are illustrated in Figure 4 and Figure 5, respectively.

5 Despite having been able to produce non-oscillatory solutions for both types of elements, it should be 6 noted that, for linear elements, in the above simulations an extremely fine mesh with an element size larger dimensions of the domain that needs to be considered. Clearly, such a simulation would require a very large number of elements, increasing the associated computational cost of the analyses.

\subsection{Effect of Péclet number on the solution to convective problems with coupled thermo-hydraulic boundary condition}

In the numerical simulation of geothermal problems, convection-dominated heat transfer may also be a significant issue (e.g. open-loop ground source energy systems), since porous media, which have a relatively low conductivity compared to other solid materials, can be subjected to a high water flow, thus resulting in a high Péclet number. To investigate the behaviour of numerical solutions to highly convective boundary value problems in geothermal engineering using the Galerkin finite element, 1D analyses of convective heat transfer along a $10 \mathrm{~m}$ soil bar, initially at a temperature of $10{ }^{\circ} \mathrm{C}$, were performed with ICFEP, using the geometry shown in Figure 1 and material properties listed in Table 1. To represent the boundary conditions of the abstraction well for an open-loop system, the coupled thermo-hydraulic boundary condition, instead of a constant temperature boundary condition of $10^{\circ} \mathrm{C}$, was prescribed at the right-hand side boundary of the mesh, while a constant temperature boundary condition of $20{ }^{\circ} \mathrm{C}$ was prescribed at the left-hand side boundary representing the injection well. Given the specified problem, thermal breakthrough should be observed when the heat front reaches the right-hand side boundary of the mesh. A constant flow velocity of $1.2 \times 10^{-4} \mathrm{~m} / \mathrm{s}$, which is of similar magnitude to the maximum velocity observed in an open-loop system with an injection/abstraction rate of $10 \mathrm{l} / \mathrm{s}$, was applied from left to right by prescribing a suitable pore water pressure gradient. The element size was varied so that different values of Péclet number could be obtained. For brevity, only results with Péclet numbers of 60 and 16 are presented, which are the maximum values of Péclet number observed in the simulations of open-loop systems presented in later sections of this paper using a coarse mesh and a fine mesh, respectively.

Figures 6 and 7 show the temperature distributions along the bar at different stages of the simulation with linear elements and a Péclet number of 60 and 16, respectively. Before thermal breakthrough, no oscillations were observed with both coarse and fine meshes. However, shortly after the heat front reached the right-hand side boundary of the 1D bar (early stage after thermal breakthrough), small oscillations of the nodal solutions were found in the coarse mesh, while using the fine mesh with the Péclet number of 16 provides spurious-free solutions throughout the analysis. Interestingly, for a Péclet number of 60 , the oscillations increase (late stage after thermal breakthrough) and finally reduce reaching a steady state with a constant uniform temperature of $20{ }^{\circ} \mathrm{C}$. The results imply that the coarse mesh with linear elements could be used for determining the time required for thermal breakthrough in open-loop systems, though it should not be used to predict the temperature distribution after this phenomenon takes place as the solution is affected by considerable oscillations. It can also be concluded from the obtained results that using the coupled thermo-hydraulic boundary conditions in simulations of highly convective problems with the Galerkin finite element method leads to non-oscillatory conditions for much larger values of Péclet number than those shown to be needed when using a prescribed temperature boundary condition. As a result, it can be concluded that an extremely fine mesh may not always be necessary in the analysis of highly convective heat transfer problems, though it should be noted that spurious oscillations still exist if an excessively large Péclet number is observed. 


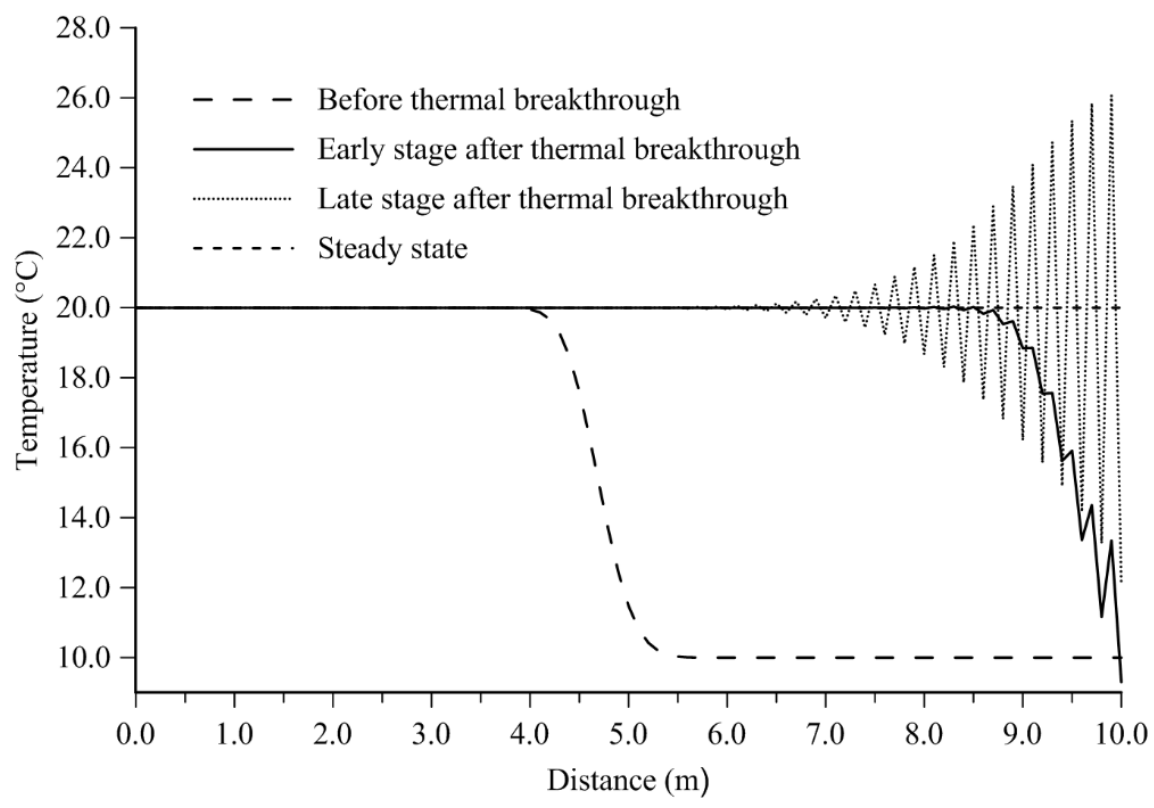

Figure 6 Temperature distribution for analysis with linear elements, coupled thermo-hydraulic boundary condition and Péclet number of 60

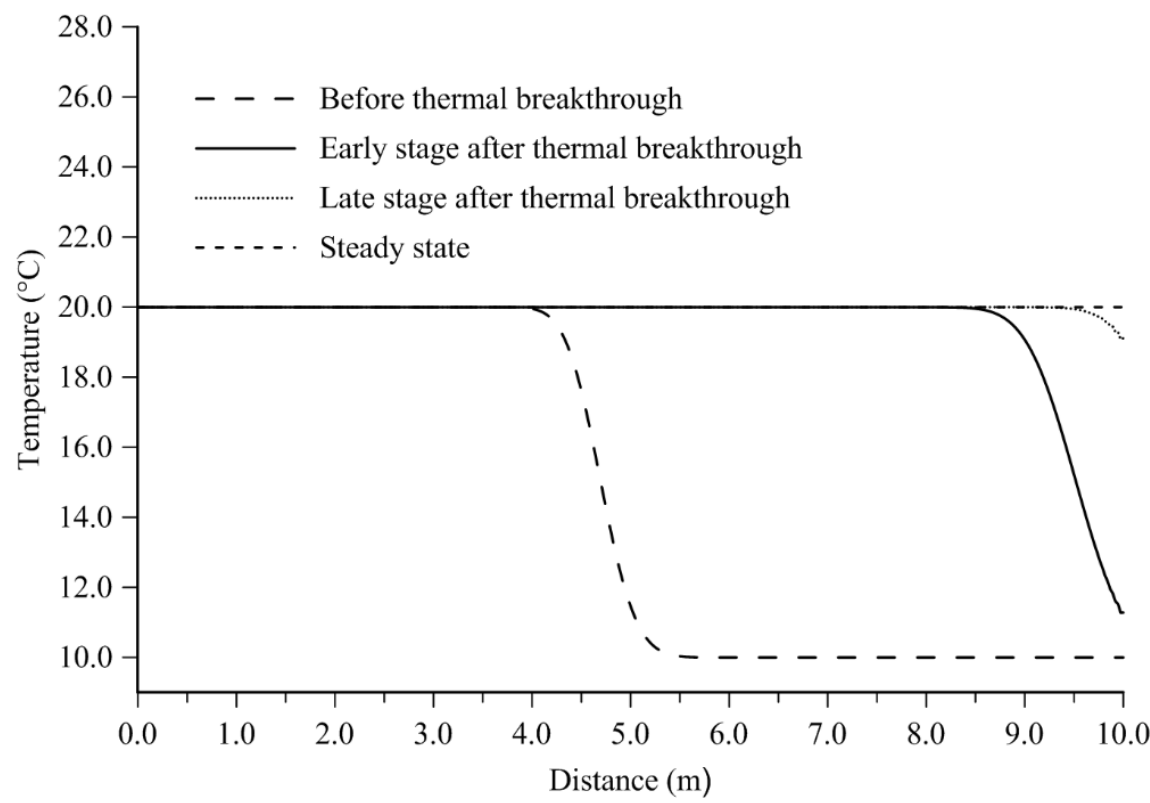

Figure 7 Temperature distribution for analysis with linear elements, coupled thermo-hydraulic boundary condition and Péclet number of 16

Figures 8 and 9 show the temperature distribution along the bar at different stages of the simulation with quadratic elements and Péclet number of 60 and 16, respectively. Compared with linear elements, quadratic elements clearly have a much better ability to deal with highly convective heat transfer with much smaller numerical oscillations being observed for identical values of Péclet number. Indeed, only slight oscillations could be observed after thermal breakthrough in the simulation with the Péclet number of 60 , suggesting that the coarse mesh with quadratic elements could be used in the simulation of open-loop systems as effectively as the fine mesh with linear elements.

It should be noted that, since the oscillation caused by using meshes with large Péclet number appear only after the heat front reaches the end of the mesh, the time taken for thermal breakthrough to occur is not affected, which has also been observed in the above exercise. 


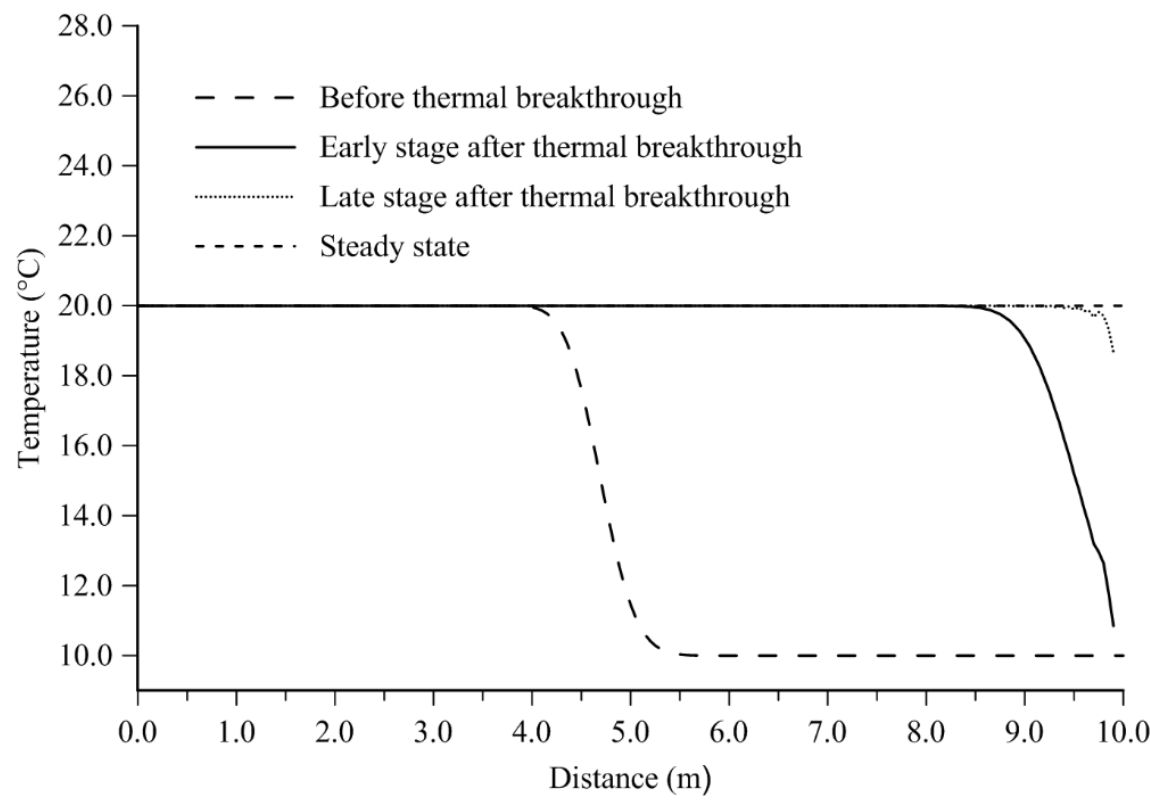

Figure 8 Temperature distribution for analysis with quadratic elements, coupled thermo-hydraulic boundary condition and Péclet number of 60

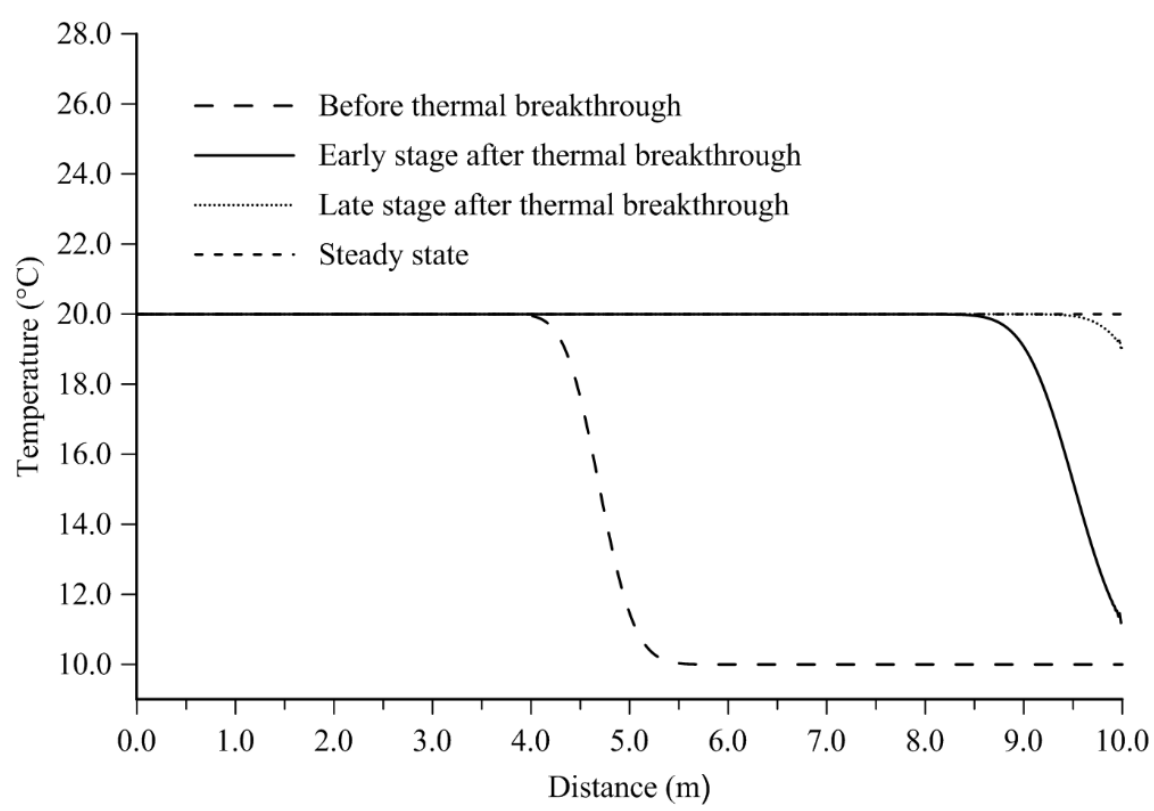

Figure 9 Temperature distribution for analysis with quadratic elements, coupled thermo-hydraulic boundary condition and Péclet number of 16

\section{Application to open-loop ground source energy systems}

\section{$8 \quad 5.1$ Problem description}

9 In order to study the performance of the coupled thermo-hydraulic facilities in ICFEP, including the 10 formulation and the boundary conditions, in a highly convective problem, a boundary value problem 11 involving an open-loop ground source energy system was chosen. In this study, the effects of element 12 size, element type as well as time-step size on the predicted time to thermal breakthrough, which, as 13 previously discussed, is the time taken for the heat front to reach the abstraction well, were 14 investigated. Predicting the time to thermal breakthrough is of great importance as its occurrence 15 affects the efficiency of the open-loop ground source energy systems and is, therefore, one of the 16 main design considerations. 
1 The simulated open-loop system (shown schematically in Figure 10) was adapted from Banks [21]. It consists of a well doublet in a 50-m thick homogenous sandstone aquifer with an initial temperature $\left(\mathrm{T}_{0}\right)$ of $10^{\circ} \mathrm{C}$, and a constant pumping rate (Q) of $10 \mathrm{l} / \mathrm{s}$. Water at a constant temperature of $20^{\circ} \mathrm{C}$ was injected into one well and the water temperature at the abstraction well $\left(\mathrm{T}_{\mathrm{a}}\right)$, which is located $20 \mathrm{~m}$ away from the injection well, was monitored. The material properties are those listed in Table 1.

\section{INJECTION WELL}

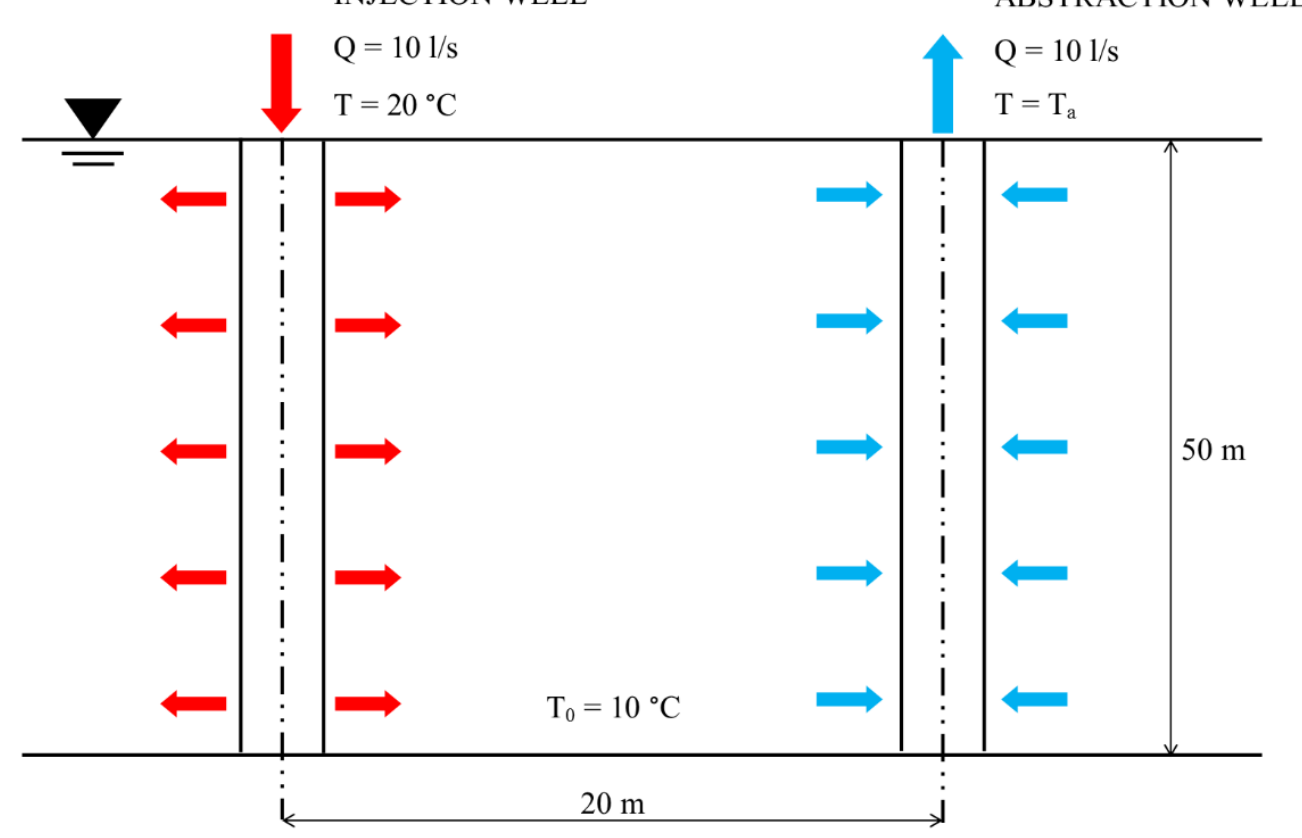

Figure 10 Schematic diagram of the well doublet system

\section{ABSTRACTION WELL}

$\mathrm{Q}=10 \mathrm{l} / \mathrm{s}$

\subsection{Numerical modelling}

Two-dimensional (2D) coupled thermo-hydraulic analyses of a horizontal cross-section through the aquifer were performed using ICFEP, where the element type, element size as well as the time-step size were varied. The aquifer was modelled as rigid in order to eliminate the mechanical coupling which is not of fundamental importance in this type of problem. The $\theta$-method was applied with the backward difference scheme $(\theta=1.0)$, which, as shown in this paper, is unconditionally stable.

Table 2 presents the details of the six analyses performed in this study. The meshes used in analyses A and $\mathrm{B}$ will be referred to as the coarse meshes, whereas the meshes used in analyses C, D, E and F will be described as the fine meshes. In all cases, the elements are smallest near the two wells, where water velocities are the highest, and the element size increases with the radial distance away from the wells. The calculated maximum Péclet number corresponds to a maximum pore water velocity of approximately $1.2 \times 10^{-4} \mathrm{~m} / \mathrm{s}$ (i.e. near the wells), and is therefore equal to 60 and 16 for the coarse mesh and the fine mesh, respectively. The contours of absolute water velocity are shown in Figure 12. It should be noted that these are the same values of Péclet number used in the one-dimensional tests presented in the previous section. Since the well doublet system is symmetric, only half of the space was discretized in order to reduce the computational effort. Figure 11 shows the coarse mesh used in analyses A and B, as an example, together with the location of the injection and abstraction wells.

The hydraulic boundary conditions applied to the mesh include injection and abstraction of water at a constant rate over the area corresponding to the wells. There is no water flow across boundaries of the mesh. A thermal boundary condition in the form of a constant temperature was prescribed at the injection well $\left(20^{\circ} \mathrm{C}\right)$, while at the far left, right and top mesh boundaries the initial temperature (10 ${ }^{\circ} \mathrm{C}$ ) was kept constant. It should be noted that since the left, right and top mesh boundaries are far away from the wells, the thermal plume cannot reach these mesh boundaries during the analysis and 
therefore, the results would not be affected by the adopted thermal boundary condition. There was no heat flux across the mesh boundary on the axis of symmetry. The coupled thermo-hydraulic boundary condition was applied where the water was leaving the mesh, i.e. at the abstraction well.

4 In the first stage of the analysis, only the hydraulic boundary conditions were activated. Once a steady 5 state water flow was established between the two wells, the thermal boundary conditions were 6 applied.

7 In a transient finite element analysis, the size of the time-step must be chosen carefully in order to find 8 a balance between accuracy and efficiency. In this paper, the effect of time-step size on the time to 9 thermal breakthrough is investigated by simulating the open-loop system using two different time-step 10 sizes: $180 \mathrm{~s}$ (analyses A, B, C and D) and $600 \mathrm{~s}$ (analyses $\mathrm{E}$ and F).

Table 2 Details of analyses performed in the study and the simulated times to thermal breakthrough ( $\left.t_{T B}\right)$

\begin{tabular}{lllllll}
\hline Analysis & $\begin{array}{l}\text { Element } \\
\text { type }\end{array}$ & $\begin{array}{l}\text { Number of } \\
\text { elements }\end{array}$ & $\begin{array}{l}\text { Min element } \\
\text { size (m) }\end{array}$ & $\begin{array}{l}\text { Max Péclet } \\
\text { number }\end{array}$ & $\begin{array}{l}\text { Time-step } \\
\text { size (s) }\end{array}$ & $\begin{array}{l}\boldsymbol{t}_{\text {TB }} \\
\text { (days) }\end{array}$ \\
\hline A & Linear & 1908 & 0.12 & 60 & 180 & 14.9 \\
B & Quadratic & 1908 & 0.12 & 60 & 180 & 14.8 \\
C & Linear & 5336 & 0.03 & 16 & 180 & 14.7 \\
D & Quadratic & 5336 & 0.03 & 16 & 180 & 14.6 \\
E & Linear & 5336 & 0.03 & 16 & 600 & 14.3 \\
F & Quadratic & 5336 & 0.03 & 16 & 600 & 14.2 \\
\hline
\end{tabular}

12

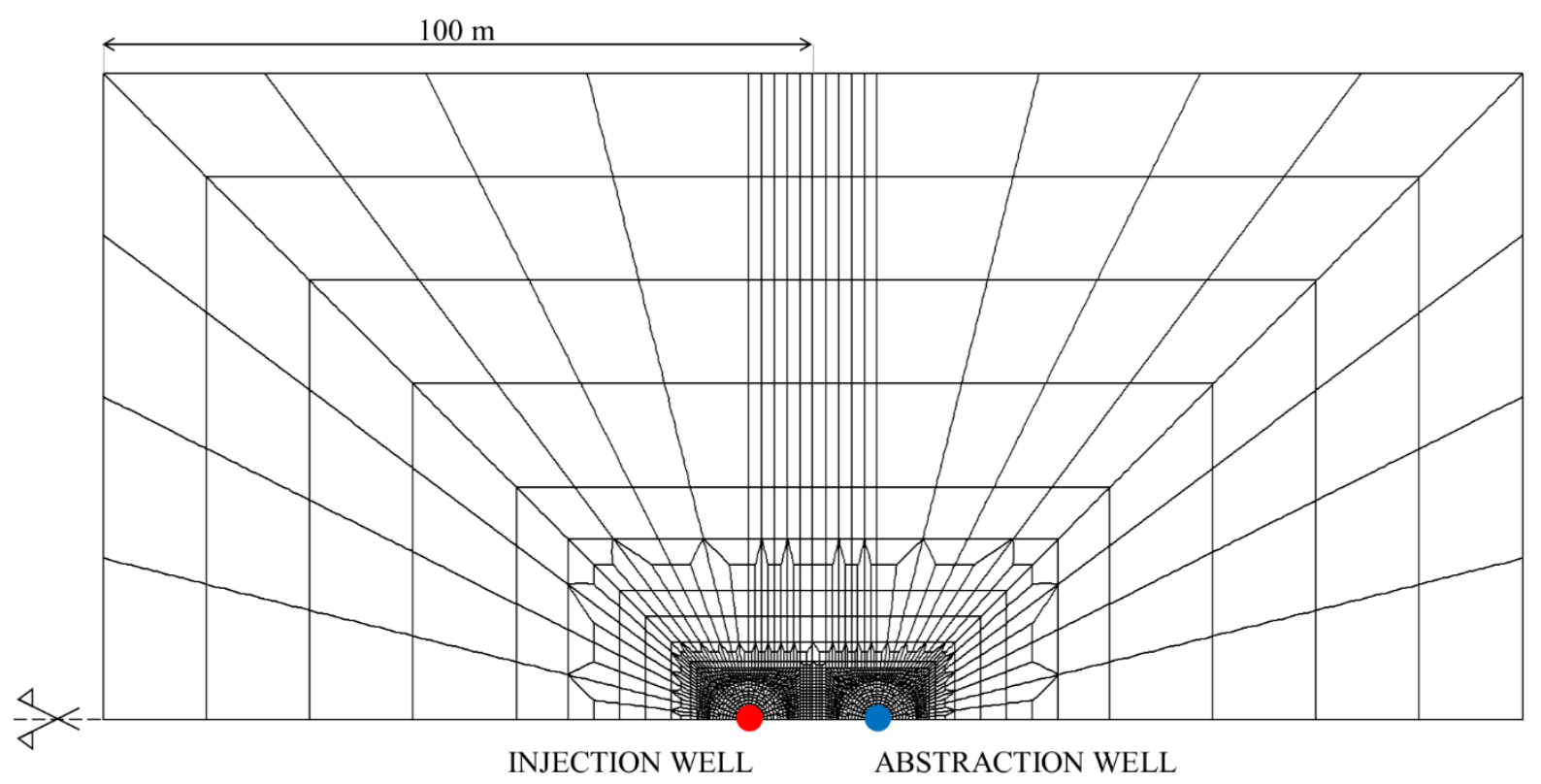




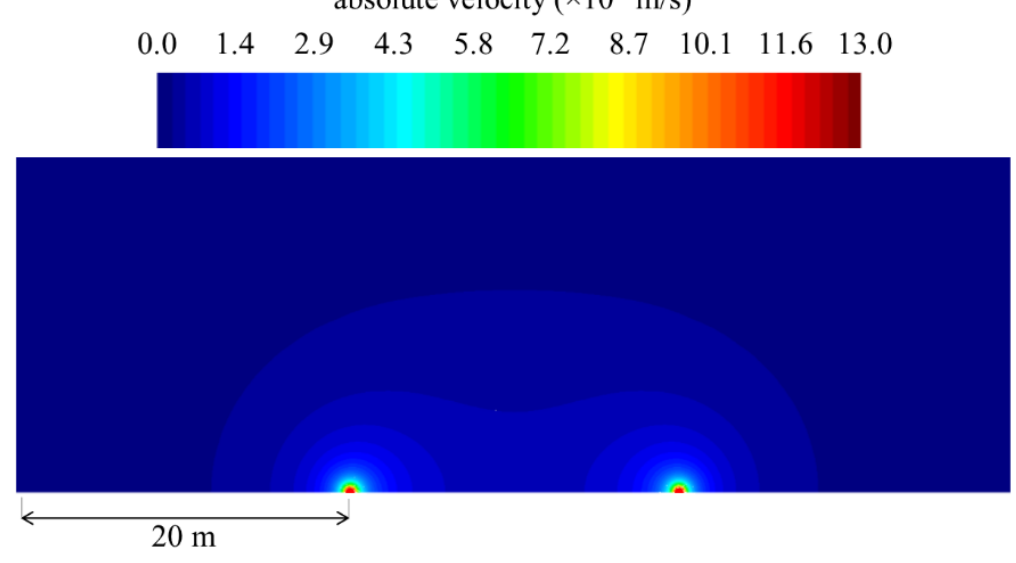

Figure 12 Contours of absolute water velocity

\section{$3 \quad 5.3$ Results}

4 In order to investigate the effect of element size, element type and the size of the time-step, six 5 analyses of the open-loop system described earlier were performed. The temperature at the abstraction 6 well was monitored and the results in terms of the time taken for thermal breakthrough to occur are 7 summarised in Table 2.

8 The series of the numerical exercises presented in the previous section have shown oscillating results 9 after thermal breakthrough for the analysis with linear elements and a Péclet number of 60 . However, 10 a non-oscillatory solution was observed before the heat front reached the end of the mesh, irrespective 11 of the element type and Péclet number. The same behaviour was observed in the simulations of the 12 open-loop system. Indeed, even though oscillations after thermal breakthrough have been registered in 13 analysis $\mathrm{A}$, the predicted time to thermal breakthrough, which is the main parameter in the design of 14 open-loop systems, was not affected. Conversely, analyses B, C, D, E and F produced non-oscillatory 15 results, and are discussed in detail in this section. As an example of the obtained results, Figure 13 16 shows temperature in the aquifer after 5, 10 and 20 days for analysis D. 

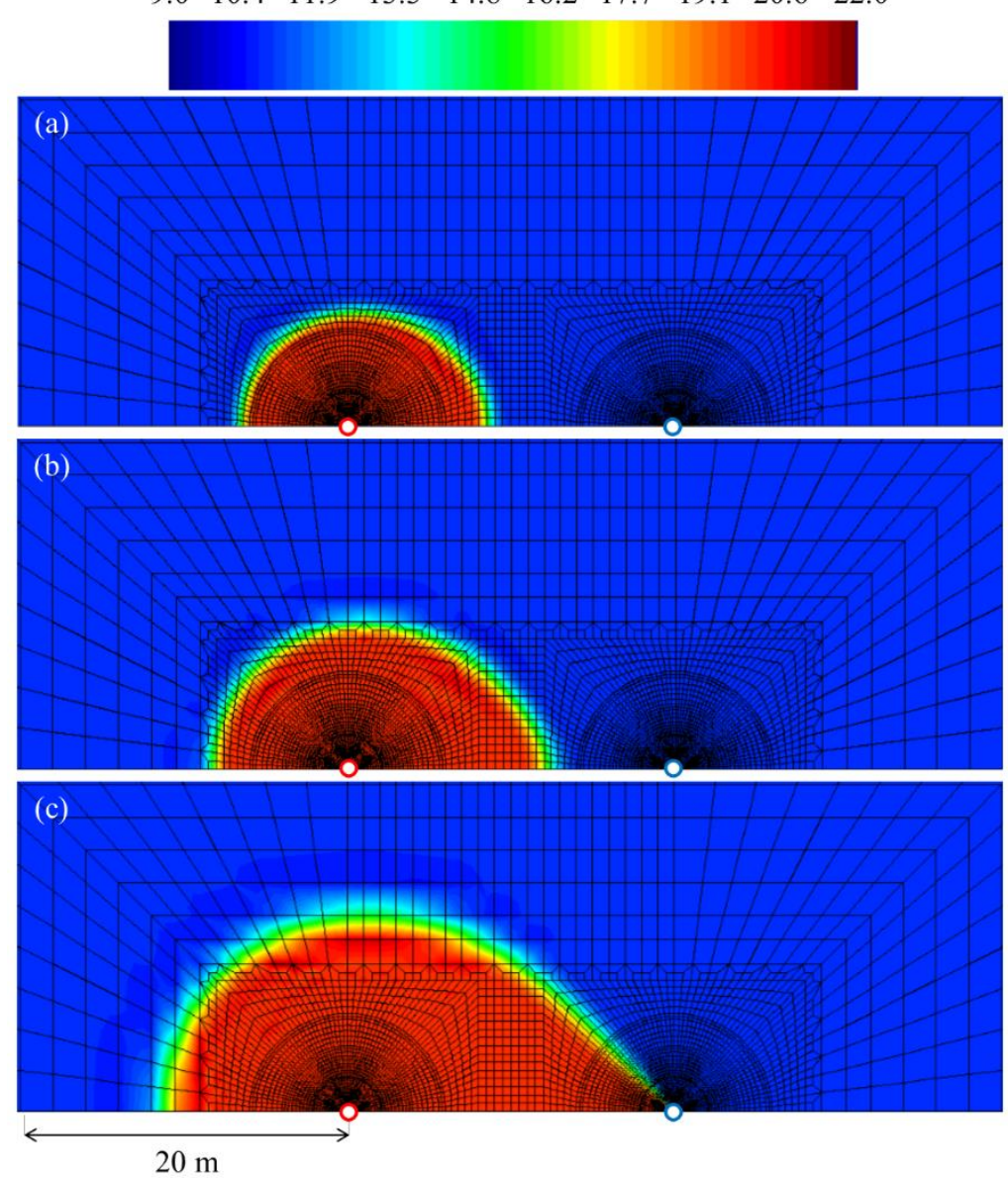

Figure 13 Aquifer temperature after (a) 5 days, (b) 10 days and (c) 20 days (analysis D)

3 The effect of element size on the time to thermal breakthrough is investigated by comparing analyses 4 B and D, both of which use quadratic elements and a time-step of $180 \mathrm{~s}$, however, the mesh in 5 analysis B is coarser than the one in analysis D. Figure 14 presents the temperature at the abstraction 6 well simulated in the two analyses. The predicted times to thermal breakthrough are 14.8 days and $7 \quad 14.6$ days for the coarse and the fine mesh, respectively. It is clear that in these cases the Péclet 8 number has very little effect on the temperature evolution at the abstraction well. A similar result may 9 be observed when comparing the analyses using linear elements (analyses A and C), where the times 10 to thermal breakthrough are 14.9 days and 14.7 days for the coarse mesh and the fine mesh, 11 respectively. 


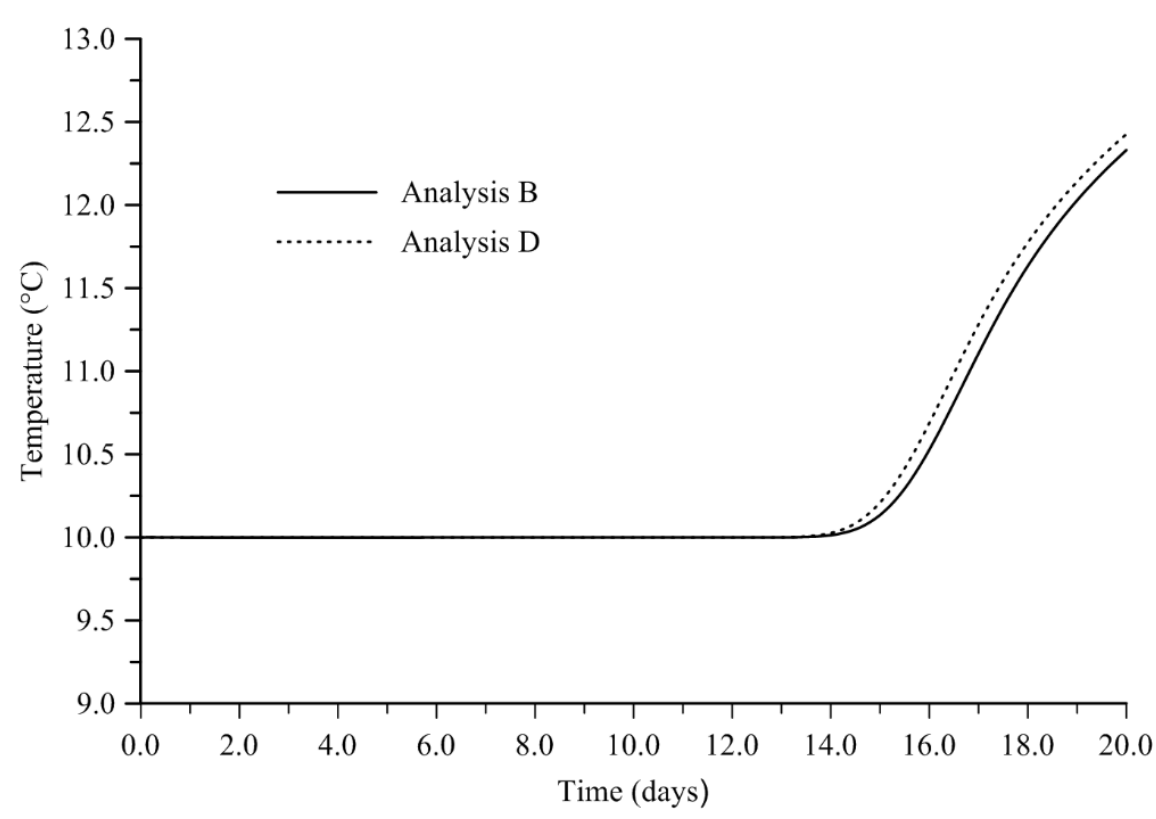

Figure 14 Effect of element size on the temperature at the abstraction well

The effect of element type on the simulation of the open-loop system is illustrated in Figure 15, where the evolutions of temperature at the abstraction well obtained using a linear mesh (analysis C) and a quadratic mesh (analysis D) are compared. Both analyses were carried out using meshes characterised by a maximum Péclet number of 16 (i.e. the fine meshes) and a time-step size of 180s. The predicted times to thermal breakthrough are 14.7 days and 14.6 days for the linear and the quadratic elements, respectively. A comparison between analyses A and B (i.e. coarse meshes) shows a similar difference (0.1 days), with the use of linear elements also leading to a longer time required for thermal breakthrough to occur. Therefore, it can be concluded that the effect of element type is also very small.

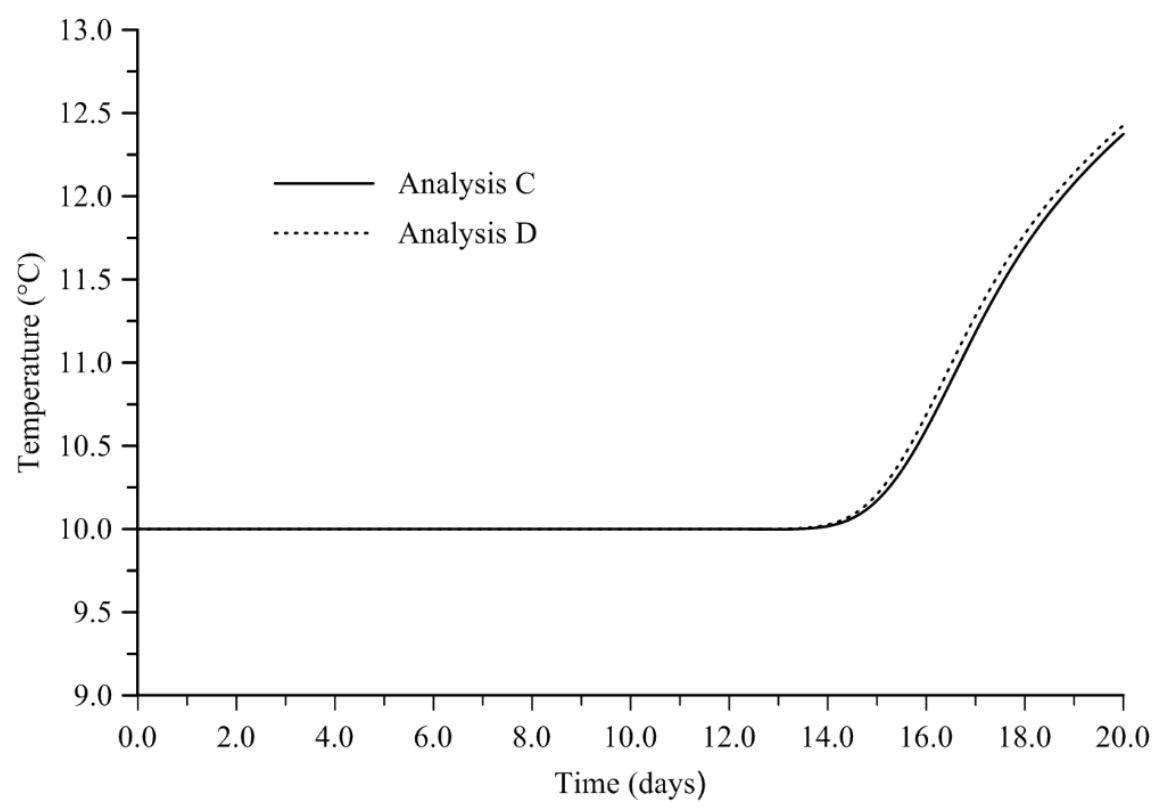

Figure 15 Effect of element type on the temperature at the abstraction well

Lastly, Figure 16 shows the effect of the size of the time-step on the temperature of the abstraction well by comparing analysis $\mathrm{D}$ with $\Delta t=180 \mathrm{~s}$ and analysis $\mathrm{F}$ with $\Delta t=600 \mathrm{~s}$, both of which use the fine mesh with quadratic elements. The times to thermal breakthrough are 14.6 days and 14.2 days for the analyses D and F, respectively. Additionally, the corresponding times for analyses $\mathrm{C}$ and $\mathrm{E}$ are 
14.7 days and 14.3 days, respectively, as listed in Table 2. A difference of 0.4 days for both cases indicates that the chosen range of time-step sizes is acceptable in terms of accuracy of the solution, and it is therefore more computationally efficient to simulate this problem with the larger time-step size.

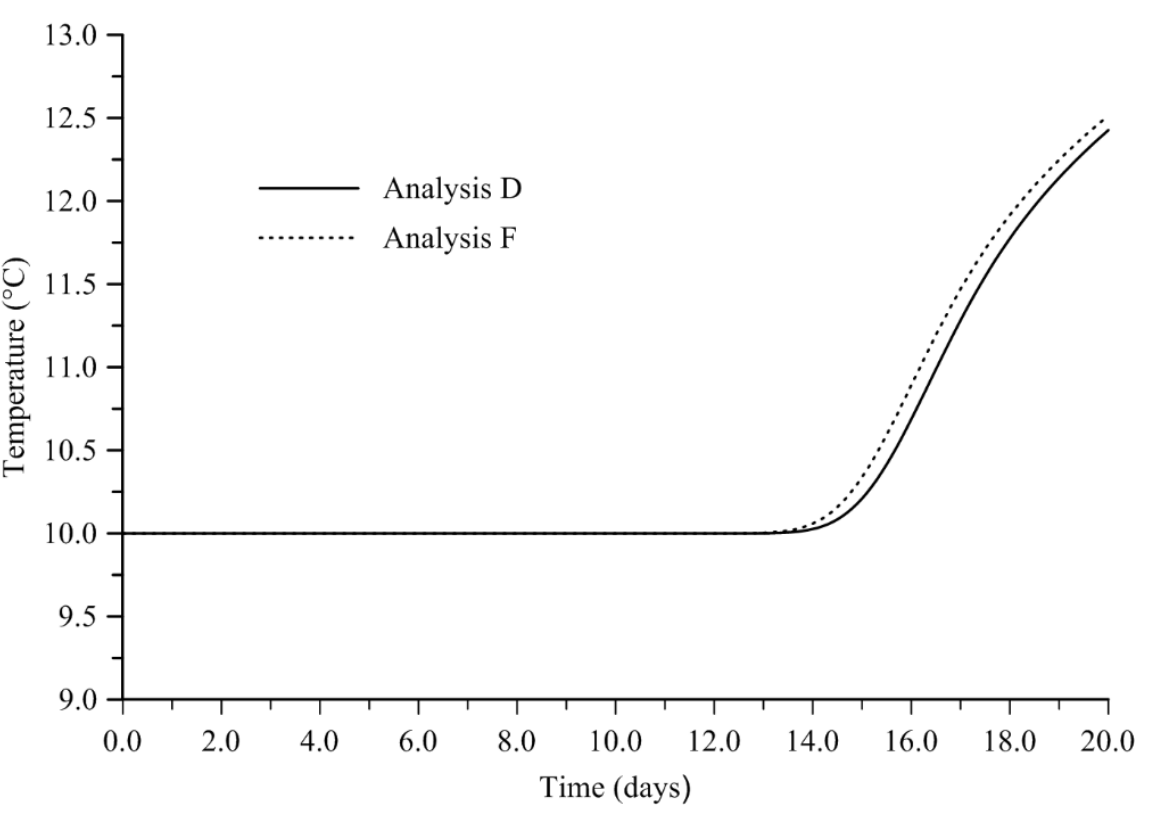

Figure 16 Effect of time-step size on the temperature at the abstraction well

Approximate solutions to the thermal plume, which estimate the time to thermal breakthrough, have been developed for various cases of well doublet systems (e.g. [21-25]). They are often based on assumptions such as [21]:

- The aquifer is homogenous and isotropic and has a constant thickness

- The wells are fully penetrating

- The problem is two-dimensional and there is no groundwater or heat flux in the out-of-plane direction

- Both the pumping rate and the injection temperature are constant

- There is an instantaneous thermal equilibrium between the porous matrix and the groundwater

Since these assumptions are identical to those behind the numerical simulations of the open-loop system presented in this section, it is possible to compare directly the computed results with one of the approximate solutions. For example, the approximate model by Lippmann and Tsang [22] and Banks $[21,25]$ predicts the time to thermal breakthrough as 15.4 days for the well doublet considered in this paper. This value agrees very well with the range of times to thermal breakthrough obtained from the six analyses (14.2 to 14.9 days), suggesting that the approximate solutions can be used for preliminary design of simple well doublet systems. However, it should be stressed that these solutions are not applicable to more complex scenarios (e.g. multiple well doublets or inhomogeneous conditions in the aquifer), and that numerical analysis is necessary in such cases.

\section{Conclusions}

This paper presents numerical facilities necessary for performing coupled thermo-hydraulic FE analysis, including the governing formulation, as well as a coupled thermo-hydraulic boundary condition. The latter prescribes a heat flux where water leaves or enters the mesh to balance the energy associated with the water flow through the boundary. These facilities were implemented in the finite element software ICFEP and validation exercises showed an excellent match between the 
1 ICFEP results and corresponding analytical solutions for heat flux through conduction and 2 convection.

3 The investigation on the stability of the $\theta$-method time integration scheme for solving the equation of 4 convective heat transfer showed that the method is unconditionally stable for $0.5 \leq \theta \leq 1$.

5 The key conclusions of the series of studies on simulating highly convective heat transfer with the 6 Galerkin finite element method (GFEM), which are presented in this paper, can be summarised as 7 follows:

8 (1) It has been demonstrated that Péclet number is a useful indicator of the significance of convective 9 heat transport and that it can be used as a measure of the element size required to avoid oscillations in 10 the results which may occur when GFEM is used.

11 (2) In cases where a prescribed temperature boundary condition is used, oscillations in nodal temperature values, which become more significant with increasing Péclet number, have been observed after the heat front reached the downstream boundary. As a result of the performed simulations, a Péclet number below 1 for linear elements, or below 2 for quadratic elements is recommended for obtaining non-oscillatory solutions.

(3) The coupled thermo-hydraulic boundary condition, which is used in the analysis of open-loop ground source energy systems, experiences oscillations at a much higher Péclet than the prescribed temperature boundary condition. In the test with linear elements and a high Péclet number, oscillations have been observed after the heat front reached the boundary. However, their amplitude was shown to reduce with time, resulting in a steady state with a constant uniform temperature. The results improve as Péclet number decreases and when quadratic elements are used.

(4) In the context of open-loop ground source energy systems these results suggest that oscillations are not expected prior to thermal breakthrough, meaning that the time needed for this phenomenon to occur predicted by the numerical analysis is not affected by the Péclet number. Indeed, this has been confirmed by the simulations of an open-loop system presented in this paper. Additionally, it has been demonstrated that the element type has no significant effect on the computed time to thermal breakthrough, with the range of results obtained from the analyses showing a good agreement with the approximate solution for the well doublet system proposed by Lippmann and Tsang [22] and Banks $[21,25]$.

However, it should be noted that oscillations in the temperature distribution after thermal breakthrough may still occur when an excessively large Péclet number is used. Naturally, this may have a very negative impact on the analysis if thermo-mechanical coupling is considered in a convection-dominated problem, meaning that, in such scenario, the element size and type must be chosen carefully.

The paper shows that the Galerkin finite element method, as implemented in ICFEP, is capable of simulating highly convective coupled thermo-hydraulic problems in geotechnical engineering, such as well doublet systems, if the mesh, the time marching scheme and the boundary conditions are chosen adequately.

\section{Acknowledgements}

40 This research is funded by China Scholarship Council (CSC) and Engineering and Physical Sciences

41 Research Council (EPSRC). 


\section{References}

[1] D. Banks, An Introduction to Thermogeology: Ground Source Heating and Cooling, WileyBlackwell, Chichester, 2012.

[2] O. Boennec, Shallow ground energy systems, Proceedings of Institution of Civil Engineers: Energy 161 (2008) 57-61.

[3] F.K. Todd, D. Banks, Modelling of a thermal plume in the Sherwook Sandstone: A case study in North Yorkshire, UK, in Proceedings Effstock 2009, Galloway, USA, 2009.

[4] C.J. Gandy, L. Clarke, D. Banks, P.L. Younger, Predictive modelling of groundwater abstraction and artificial recharge of cooling water, Quarterly Journal of Engineering Geology and Hydrogeology 43 (2010) 279-288.

[5] C. Clauser, Numerical Simulation of Reactive Flow in Hot Aquifers: SHEMAT and Processing SHEMAT, Springer, Berlin, 2003.

[6] H.J. Diersch, FEFLOW: Finite Element Modeling of Flow, Mass and Heat Transport in Porous and Fractured Media, Springer, Berlin, 2014.

[7] S. Lo Russo, M.V. Civita, Open-loop groundwater heat pumps development for large buildings: A case study, Geothermics 38 (2009) 335-345.

[8] Y. Nam, R. Ooka, Numerical simulation of ground heat and water transfer for groundwater heat pump system based on real-scale experiment, Energy and Buildings 42 (2010) 69-75.

[9] D.W. Bridger, D.M. Allen, Heat transport simulations in a heterogeneous aquifer used for aquifer thermal energy storage (ATES), Canadian Geotechnical Journal 47 (2010) 96-115.

[10] J.C. Heinrich, P.S. Huyakorn, O.C. Zienkiewicz, A.R. Mitchell, An 'upwind' finite element scheme for two-dimensional convective transport equation, International Journal for Numerical Methods in Engineering 11 (1977) 131-143.

[11] J.C. Heinrich, O.C. Zienkiewicz, Quadratic finite element schemes for two-dimensional convective-transport problems, International Journal for Numerical Methods in Engineering 11 (1977) 1831-1844.

[12] T.J.R. Hughes, L.P. Franca, G.M. Hulbert, A new finite element formulation for computational fluid dynamics: VIII. The galerkin/least-squares method for advectivediffusive equations, Computer Methods in Applied Mechanics and Engineering 73 (1989) 173-189.

[13] C. Johnson, A. Szepessy, P. Hansbo, On the convergence of shock-capturing streamline diffusion finite element methods for hyperbolic conservation laws, Mathematics of Computation 54 (1990) 107-129.

[14] D.M. Potts, L. Zdravković, Finite Element Analysis in Geotechnical Engineering: Theory, Thomas Telford, London, 1999.

[15] O.T. Farouki, Thermal Properties of Soils, United States Army Corps of Engineers, Cold Regions Research and Engineering Laboratory, Hanover, 1981.

[16] M.T. van Genuchten, W.J. Alves, Analytical solutions of the one-dimensional convectivedispersive solute transport equation, Technical Bulletin Number 1661, U.S. Department of Agriculture, Agricultural Research Service, 1982.

[17] O.C. Zienkiewicz, R.L. Taylor, J.Z. Zhu, The finite element method: Its basis and fundamentals, Elsevier, Waltham, 2005.

[18] J.R. Booker, J.C. Small, An investigation of the stability of numerical solutions of Biot's equations of consolidation, International Journal of Solids and Structures 11 (1975) 907-917.

[19] R. Al-Khoury, Computational Modeling of Shallow Geothermal Systems, Taylor \& Francis, Boca Raton, 2012.

[20] J. Donea, A. Huerta, Finite Element Methods for Flow Problems, John Wiley \& Sons, Chichester, 2003.

[21] D. Banks, The application of analytical solutions to the thermal plume from a well doublet ground source heating or cooling scheme, Quarterly Journal of Engineering Geology and Hydrogeology 44 (2011) 191-197.

[22] M.J. Lippmann, C.F. Tsang, Ground-Water Use for Cooling: Associated Aquifer Temperature Changes, Ground Water 18 (1980) 452-458. 
[23] C. Clyde, G. Madabhushi, Spacing of Wells for Heat Pumps, Journal of Water Resources Planning and Management 109 (1983) 203-212.

[24] J. Luo, P.K. Kitanidis, Fluid residence times within a recirculation zone created by an extraction-injection well pair, Journal of Hydrology 295 (2004) 149-162.

[25] D. Banks, Thermogeological assessment of open-loop well-doublet schemes: a review and synthesis of analytical approaches, Hydrogeology Journal 17 (2009) 1149-1155. 